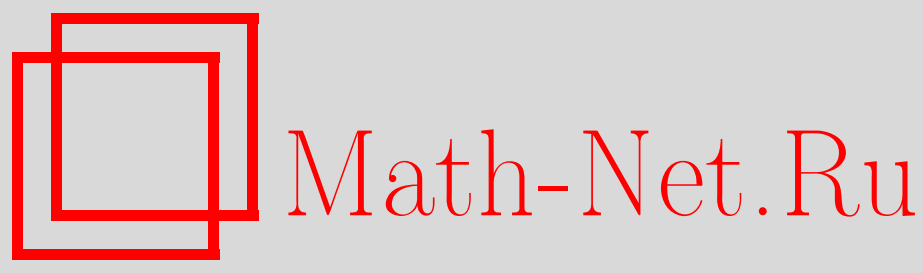

Т. Х. Расулов, О ветвях существенного спектра решетчатой модели спин-бозон с не более чем двумя фотонами, TMФ, 2016, том 186, номер 2, 293-310

DOI: https://doi.org/10.4213/tmf8854

Использование Общероссийского математического портала Math-Net.Ru подразумевает, что вы прочитали и согласны с пользовательским соглашением http: //www . mathnet.ru/rus/agreement

Параметры загрузки:

IP : 54.80 .73 .141

26 апреля 2023 г., 14:53:22

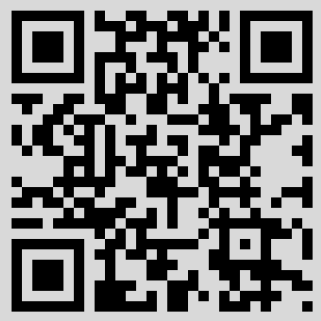




\title{
О ВЕТВЯХ СУЩЕСТВЕННОГО СПЕКТРА РЕШЕТЧАТОЙ МОДЕЛИ СПИН-БОЗОН С НЕ БОЛЕЕ ЧЕМ ДВУМЯ ФОТОНАМИ
}

\begin{abstract}
Рассматривается решетчатый аналог $\mathcal{A}_{m}$ модели светового излучения с неподвижным атомом и не более чем $m$ фотонами $(m=1,2)$. Описано местоположение существенного спектра оператора $\mathcal{A}_{2}$ через спектр оператора $\mathcal{A}_{1}$, т. е. выделены двухчастичная и трехчастичная ветви существенного спектра оператора $\mathcal{A}_{2}$. Доказано, что существенный спектр состоит из объединения не более чем шести отрезков, и изучено расположение этих отрезков. Получены оценки для нижних граней двухчастичных и трехчастичных ветвей.
\end{abstract}

Ключевые слова: модель спин-бозон, бозонное пространство Фока, блочно-операторная матрица, операторы рождения и уничтожения, существенный и дискретный спектры, критерий Вейля.

DOI: $10.4213 / \operatorname{tmf} 8854$

\section{1. ВВЕДЕНИЕ И ПОСТАНОВКА ЗАДАЧИ}

Блочно-операторная матрица - это матрица, элементы которой являются линейными операторами в банаховом или гильбертовом пространстве [1]. Один из специальных классов блочно-операторных матриц составляют гамильтонианы системы с несохраняющимся числом квантовых частиц на целочисленной решетке. Их количество может быть неограниченным, как в случае моделей спин-бозон [2], [3], или ограниченным, как в случае "урезанных" моделей спин-бозон [4], [5]. Отметим, что такие системы обычно возникают в задачах физики твердого тела [6], квантовой теории поля [7], статистической физики [8], магнитогидродинамики [9] и квантовой механики [10].

В хорошо известной модели светового излучения (так называемой модели спинбозон, см. статьи [2]-[5]) предполагается, что атом, который может находиться в двух

Работа поддержана программой Einstein Foundation, Berlin, Germany.

* Физико-математический факультет, Бухарский государственный университет, Бухара, Узбекистан. E-mail: rth@mail.ru 
состояниях - основном с энергией $-\varepsilon$ и возбужденном с энергией $\varepsilon$, испускает и поглощает фотоны, переходя из одного состояния в другое. Решетчатый аналог этой модели задается (формальным) выражением

$$
\mathcal{A}:=\varepsilon \sigma_{z}+\int_{\mathbb{T}^{d}} w(k) a^{*}(k) a(k) d k+\alpha \sigma_{x} \int_{\mathbb{T}^{d}} v(k)\left(a^{*}(k)+a(k)\right) d k
$$

и действует в гильбертовом пространстве $\mathcal{L}:=\mathbb{C}^{2} \otimes \mathcal{F}_{\mathrm{b}}\left(L_{2}\left(\mathbb{T}^{d}\right)\right)$, состоящем из последовательностей

$$
F=\left\{f_{0}^{(s)}, f_{1}^{(s)}\left(k_{1}\right), f_{2}^{(s)}\left(k_{1}, k_{2}\right), \ldots, f_{n}^{(s)}\left(k_{1}, \ldots, k_{n}\right), \ldots ; s= \pm\right\}
$$

функций возрастающего числа переменных $\left(k_{1}, \ldots, k_{n}\right), k_{i} \in \mathbb{T}^{d}$, и дискретной переменной $s= \pm$, симметричных по переменным $k_{i}, i=1, \ldots, n, n \in \mathbb{N}$. Здесь $L_{2}\left(\mathbb{T}^{d}\right)-$ гильбертово пространство квадратично-интегрируемых функций, определенных на $d$-мерном торе $\mathbb{T}^{d}, \mathcal{F}_{\mathrm{b}}\left(L_{2}\left(\mathbb{T}^{d}\right)\right)$ - стандартное бозонное пространство Фока над пространством $L_{2}\left(\mathbb{T}^{d}\right)$ :

$$
\mathcal{F}_{\mathrm{b}}\left(L_{2}\left(\mathbb{T}^{d}\right)\right):=\mathbb{C} \oplus L_{2}\left(\mathbb{T}^{d}\right) \oplus L_{2}^{\mathrm{sym}}\left(\left(\mathbb{T}^{d}\right)^{2}\right) \oplus \cdots,
$$

$L_{2}^{\text {sym }}\left(\left(\mathbb{T}^{d}\right)^{n}\right)$ - гильбертово пространство симметричных функций $n$ переменных, определенных на $\left(\mathbb{T}^{d}\right)^{n}, n \geqslant 2$. В формуле $(1)$ операторы $a^{*}(k)$ и $a(k)$ суть операторы рождения-уничтожения, $\varepsilon>0$,

$$
\sigma_{z}:=\left(\begin{array}{cc}
1 & 0 \\
0 & -1
\end{array}\right), \quad \sigma_{x}:=\left(\begin{array}{cc}
0 & 1 \\
1 & 0
\end{array}\right)
$$

суть матрицы Паули, $w(k)$ - энергия фотона с импульсом $k, v(\cdot)$ - вещественнозначная непрерывная функция на $\mathbb{T}^{d}, \alpha>0$ - параметр взаимодействия. При этом $w(\cdot)$ есть вещественнозначная непрерывная функция на $\mathbb{T}^{d}$ и $\min _{k \in \mathbb{T}^{d}} w(k)=0$.

Задача о полном спектральном описании оператора $\mathcal{A}$ является довольно трудной. В связи с этим естественно рассмотреть упрощенные ("урезанные") модели, отличающиеся от описанной выше модели тем, что возможное число фотонов в них ограничено и не превосходит $m, m \in \mathbb{N}$. Гильбертовым пространством состояний такой модели служит пространство

$$
\mathcal{L}_{m}:=\mathbb{C}^{2} \otimes \mathcal{F}_{\mathrm{b}}^{(m)}\left(L_{2}\left(\mathbb{T}^{d}\right)\right)
$$

где

$$
\begin{aligned}
& \mathcal{F}_{\mathrm{b}}^{(1)}\left(L_{2}\left(\mathbb{T}^{d}\right)\right):=\mathbb{C} \oplus L_{2}\left(\mathbb{T}^{d}\right), \\
& \mathcal{F}_{\mathrm{b}}^{(m)}\left(L_{2}\left(\mathbb{T}^{d}\right)\right):=\mathbb{C} \oplus L_{2}\left(\mathbb{T}^{d}\right) \oplus L_{2}^{\mathrm{sym}}\left(\left(\mathbb{T}^{d}\right)^{2}\right) \oplus \cdots \oplus L_{2}^{\mathrm{sym}}\left(\left(\mathbb{T}^{d}\right)^{m}\right), \quad m \geqslant 2 .
\end{aligned}
$$

Оператор $\mathcal{A}_{m}$ в гильбертовом пространстве $\mathcal{L}_{m}$ задается формулой

$$
\mathcal{A}_{m}:=P_{\mathcal{L}_{m}} \mathcal{A} P_{\mathcal{L}_{m}},
$$

где $P_{\mathcal{L}_{m}}$ - проектор в пространстве $\mathcal{L}$ на подпространство $\mathcal{L}_{m}$, а гамильтониан $\mathcal{A}$ определен выражением (1).

В работах [2], [3] предпринято серьезное математическое исследование спектральных свойств "непрерывного" (стандартного) гамильтониана модели спин-бозон. В работе [4] изучена модель светового излучения с неподвижным атомом и не более 
чем тремя фотонами $(m=3)$ в “непрерывном" случае, т. е. в бозонном фоковском пространстве над $L_{2}\left(\mathbb{R}^{d}, \mathbb{C}^{2}\right)$. В [4] проведен спектральный анализ гамильтониана с помощью теории рассеяния в паре пространств со специально выбранным вложением. В частности, доказаны существование волновых операторов и их асимптотическая полнота. При этом все построения опираются на детальный анализ резольвенты. В работе [5] полностью изучены случаи $m=1,2$ при малых значениях параметра $\alpha$. Отметим, что в работах [2]-[5] условия малости параметра взаимодействия $\alpha$ существенны.

В настоящей работе мы рассмотрим случай $m=2$. При этом оператор $\mathcal{A}_{2}=$ $P_{\mathcal{L}_{2}} \mathcal{A} P_{\mathcal{L}_{2}}$ действует в гильбертовом пространстве $\mathcal{L}_{2}$ и записывается как трехдиагональная блочно-операторная матрица размера $3 \times 3$

$$
\mathcal{A}_{2}:=\left(\begin{array}{ccc}
\mathcal{A}_{00} & \mathcal{A}_{01} & 0 \\
\mathcal{A}_{01}^{*} & \mathcal{A}_{11} & \mathcal{A}_{12} \\
0 & \mathcal{A}_{12}^{*} & \mathcal{A}_{22}
\end{array}\right)
$$

с матричными элементами

$$
\begin{gathered}
\mathcal{A}_{00} f_{0}^{(s)}=s \varepsilon f_{0}^{(s)}, \quad \mathcal{A}_{01} f_{1}^{(s)}=\alpha \int_{\mathbb{T}^{d}} v(t) f_{1}^{(-s)}(t) d t \\
\left(\mathcal{A}_{11} f_{1}^{(s)}\right)(p)=(s \varepsilon+w(p)) f_{1}^{(s)}(p), \quad\left(\mathcal{A}_{12} f_{2}^{(s)}\right)(p)=\alpha \int_{\mathbb{T}^{d}} v(t) f_{2}^{(-s)}(p, t) d t, \\
\left(\mathcal{A}_{22} f_{2}^{(s)}\right)(p, q)=(s \varepsilon+w(p)+w(q)) f_{2}^{(s)}(p, q),
\end{gathered}
$$

где $\left\{f_{0}^{(s)}, f_{1}^{(s)}, f_{2}^{(s)}, s= \pm\right\} \in \mathcal{L}_{2}$. Здесь $\mathcal{A}_{i j}^{*}$ - оператор, сопряженный с $\mathcal{A}_{i j}, i<j$, а норма элемента

$$
F=\left\{f_{0}^{(s)}, f_{1}^{(s)}, f_{2}^{(s)}, s= \pm\right\} \in \mathcal{L}_{2}
$$

задается выражением

$$
\|F\|^{2}=\sum_{s= \pm}\left(\left|f_{0}^{(s)}\right|^{2}+\int_{\mathbb{T}^{d}}\left|f_{1}^{(s)}(p)\right|^{2} d p+\frac{1}{2} \int_{\left(\mathbb{T}^{d}\right)^{2}}\left|f_{2}^{(s)}(p, q)\right|^{2} d p d q\right) .
$$

Хотя решетчатая модель $\mathcal{A}_{2}$ ограничена и самосопряжена, ее спектр имеет более сложный характер по сравнению с непрерывным случаем. Следует отметить, что в непрерывном случае двухчастичная и трехчастичная ветви существенного спектра состоят из полуосей $[\kappa, \infty)$, где $\kappa<0$, и пересекаются. Следовательно (см. статью [5]), для описания существенного спектра этой модели достаточно найти собственные значения в случае $m=1$, а используемая при этом техника элементарна. В решетчатом случае двухчастичная и трехчастичная ветви существенного спектра состоят из отрезков конечной длины, причем они могут не пересекаться. Возникает вопрос: существуют ли собственные значения в лакунах и, если существуют, конечно ли их число? Поэтому изучение существенного спектра и условий существования лакун существенного спектра оператора $\mathcal{A}_{2}$ является важной задачей для дальнейшего исследования спектральных свойств рассматриваемого оператора.

Следует отметить, что некоторые спектральные свойства модели $\mathcal{A}_{2}$ ранее изучены в работе [11]. В частности, в ней описано (без доказательства) местоположение существенного спектра оператора $\mathcal{A}_{2}$ и получена оценка для нижней грани существенного спектра в случае $d=1$. В настоящей работе проведено детальное 
исследование существенного спектра оператора $\mathcal{A}_{2}$ при любых значениях размерности $d \in \mathbb{N}$ и параметра взаимодействия $\alpha>0$. В дополнение к уже известным свойствам спектра модели $\mathcal{A}_{2}$ изучена его геометрия и строго установлена его ветвистая структура. Ради удобства читателей мы приводим полное доказательство полученных результатов.

Целью настоящей работы является решение следующих задач:

- определить количество и местоположение собственных значений, а также исследовать соответствующие собственные векторы оператора $\mathcal{A}_{1}$; показать отсутствие собственных значений, лежащих в лакуне (т. е. между ветвями) существенного спектра; доказать существование собственного значения, лежащего в существенном спектре (т. е. вложенных собственных значений);

- описать местоположение существенного спектра оператора $\mathcal{A}_{2}$ и доказать, что существенный спектр этого оператора состоит из объединения не более чем шести отрезков;

- изучить структуру существенного спектра оператора $\mathcal{A}_{2}$ и оценить его нижнюю грань.

В следующих разделах мы подробно обсудим эти вопросы.

\section{2. ОПИСАНИЕ СПЕКТРА ОПЕРАТОРОВ $\mathcal{A}_{m}, m=1,2$}

В этом разделе мы сведем изучение спектра оператора $\mathcal{A}_{m}$ к изучению спектра более простого оператора $\mathcal{A}_{m}^{(s)}, s= \pm$, используя оператор перестановки, и затем опишем спектр оператора $\mathcal{A}_{m}$ через спектр оператора $\mathcal{A}_{m}^{(s)}, s= \pm$.

Сначала отметим, что оператор $\mathcal{A}_{1}$ в гильбертовом пространстве $\mathcal{F}_{\mathrm{b}}^{(1)}\left(L_{2}\left(\mathbb{T}^{d}\right)\right)$ записывается как блочно-операторная матрица размера $2 \times 2$ :

$$
\mathcal{A}_{1}:=\left(\begin{array}{cc}
\mathcal{A}_{00} & \mathcal{A}_{01} \\
\mathcal{A}_{01}^{*} & \mathcal{A}_{11}
\end{array}\right) .
$$

Пусть $m=1,2$. С целью изучения спектральных свойств оператора $\mathcal{A}_{m}$ рассмотрим также ограниченные самосопряженные операторы $\mathcal{A}_{m}^{(s)}, s= \pm$, действующие в $\mathcal{F}_{\mathrm{b}}^{(m)}\left(L_{2}\left(\mathbb{T}^{d}\right)\right)$ как блочно-операторные матрицы размера $(m+1) \times(m+1)$ :

$$
\mathcal{A}_{1}^{(s)}:=\left(\begin{array}{cc}
\widehat{\mathcal{A}}_{00}^{(s)} & \widehat{\mathcal{A}}_{01} \\
\widehat{\mathcal{A}}_{01}^{*} & \widehat{\mathcal{A}}_{11}^{(s)}
\end{array}\right), \quad \mathcal{A}_{2}^{(s)}:=\left(\begin{array}{ccc}
\widehat{\mathcal{A}}_{00}^{(s)} & \widehat{\mathcal{A}}_{01} & 0 \\
\widehat{\mathcal{A}}_{01}^{*} & \widehat{\mathcal{A}}_{11}^{(s)} & \widehat{\mathcal{A}}_{12} \\
0 & \widehat{\mathcal{A}}_{12}^{*} & \widehat{\mathcal{A}}_{22}^{(s)}
\end{array}\right)
$$

с элементами

$$
\begin{gathered}
\widehat{\mathcal{A}}_{00}^{(s)} f_{0}=s \varepsilon f_{0}, \quad \widehat{\mathcal{A}}_{01} f_{1}=\alpha \int_{\mathbb{T}^{d}} v(t) f_{1}(t) d t \\
\left(\widehat{\mathcal{A}}_{11}^{(s)} f_{1}\right)(p)=(-s \varepsilon+w(p)) f_{1}(p), \quad\left(\widehat{\mathcal{A}}_{12} f_{2}\right)(p)=\alpha \int_{\mathbb{T}^{d}} v(t) f_{2}(p, t) d t, \\
\left(\widehat{\mathcal{A}}_{22}^{(s)} f_{2}\right)(p, q)=(s \varepsilon+w(p)+w(q)) f_{2}(p, q),
\end{gathered}
$$

где

При этом

$$
\left(f_{0}, f_{1}\right) \in \mathcal{F}_{\mathrm{b}}^{(1)}\left(L_{2}\left(\mathbb{T}^{d}\right)\right), \quad\left(f_{0}, f_{1}, f_{2}\right) \in \mathcal{F}_{\mathrm{b}}^{(2)}\left(L_{2}\left(\mathbb{T}^{d}\right)\right)
$$

$$
\begin{aligned}
\left(\widehat{\mathcal{A}}_{01}^{*} f_{0}\right)(p) & =\alpha v(p) f_{0}, \\
\left(\widehat{\mathcal{A}}_{12}^{*} f_{1}\right)(p, q) & =\alpha\left(v(p) f_{1}(q)+v(q) f_{1}(p)\right), \quad\left(f_{0}, f_{1}\right) \in \mathcal{F}_{\mathrm{b}}^{(1)}\left(L_{2}\left(\mathbb{T}^{d}\right)\right) .
\end{aligned}
$$


Операторы $\widehat{\mathcal{A}}_{01}$ и $\widehat{\mathcal{A}}_{12}$ называются операторами уничтожения, а $\widehat{\mathcal{A}}_{01}^{*}$ и $\widehat{\mathcal{A}}_{12}^{*}$ - операторами рождения [7]. Оператор уничтожения снижает количество частиц в данном состоянии на единицу, а оператор рождения увеличивает число частиц в данном состоянии на единицу и является сопряженным к оператору уничтожения. Такие операторы имеют широкое применение в квантовой механике, в частности при изучении квантовых гармонических осцилляторов и систем многих частиц [12].

Несмотря на то что аналог модели $\mathcal{A}_{2}^{(s)}$ изучен во многих работах (см., например, статьи [13]-[15]), введенное выше определение позволяет получить более точную информацию о существенном спектре оператора $\mathcal{A}_{2}^{(s)}$. Далее $\sigma(\cdot), \sigma_{\mathrm{ess}}(\cdot), \sigma_{\mathrm{p}}(\cdot)$ и $\sigma_{\text {disc }}(\cdot)$ суть спектр, существенный спектр, точечный спектр и дискретный спектр ограниченного самосопряженного оператора соответственно.

Установим связь между спектрами операторов $\mathcal{A}_{m}$ и $\mathcal{A}_{m}^{(s)}, s= \pm$.

Лемма 1. Пусть $m=1,2$. Имеет место равенство $\sigma\left(\mathcal{A}_{m}\right)=\sigma\left(\mathcal{A}_{m}^{(+)}\right) \cup \sigma\left(\mathcal{A}_{m}^{(-)}\right)$. Более того,

$$
\sigma_{\text {ess }}\left(\mathcal{A}_{m}\right)=\sigma_{\text {ess }}\left(\mathcal{A}_{m}^{(+)}\right) \cup \sigma_{\mathrm{ess}}\left(\mathcal{A}_{m}^{(-)}\right), \quad \sigma_{\mathrm{p}}\left(\mathcal{A}_{m}\right)=\sigma_{\mathrm{p}}\left(\mathcal{A}_{m}^{(+)}\right) \cup \sigma_{\mathrm{p}}\left(\mathcal{A}_{m}^{(-)}\right) .
$$

ДоказАтЕЛьство. Введем два оператора перестановки $\Phi_{m}, m=1,2$ :

$$
\begin{gathered}
\Phi_{m}: \mathcal{L}_{m} \rightarrow \mathcal{F}_{\mathrm{b}}^{(m)}\left(L_{2}\left(\mathbb{T}^{d}\right)\right) \oplus \mathcal{F}_{\mathrm{b}}^{(m)}\left(L_{2}\left(\mathbb{T}^{d}\right)\right), \quad m=1,2, \\
\Phi_{1}:\left(f_{0}^{(+)}, f_{0}^{(-)}, f_{1}^{(+)}, f_{1}^{(-)}\right) \rightarrow\left(f_{0}^{(+)}, f_{1}^{(-)}, f_{0}^{(-)}, f_{1}^{(+)}\right), \\
\Phi_{2}:\left(f_{0}^{(+)}, f_{0}^{(-)}, f_{1}^{(+)}, f_{1}^{(-)}, f_{2}^{(+)}, f_{2}^{(-)}\right) \rightarrow\left(f_{0}^{(+)}, f_{1}^{(-)}, f_{2}^{(+)}, f_{0}^{(-)}, f_{1}^{(+)}, f_{2}^{(-)}\right) .
\end{gathered}
$$

Очевидно, что $\Phi_{m}-$ унитарный оператор и

$$
\begin{gathered}
\Phi_{m}^{-1}: \mathcal{F}_{\mathrm{b}}^{(m)}\left(L_{2}\left(\mathbb{T}^{d}\right)\right) \oplus \mathcal{F}_{\mathrm{b}}^{(m)}\left(L_{2}\left(\mathbb{T}^{d}\right)\right) \rightarrow \mathcal{L}_{m}, \quad m=1,2, \\
\Phi_{1}^{-1}:\left(\phi, \phi^{\prime}\right) \rightarrow\left(\phi_{0}, \phi_{0}^{\prime}, \phi_{1}^{\prime}, \phi_{1}\right), \\
\phi=\left(\phi_{0}, \phi_{1}\right), \quad \phi^{\prime}=\left(\phi_{0}^{\prime}, \phi_{1}^{\prime}\right) \in \mathcal{F}_{\mathrm{b}}^{(1)}\left(L_{2}\left(\mathbb{T}^{d}\right)\right), \\
\Phi_{2}^{-1}:\left(\varphi, \varphi^{\prime}\right) \rightarrow\left(\varphi_{0}, \varphi_{0}^{\prime}, \varphi_{1}^{\prime}, \varphi_{1}, \varphi_{2}, \varphi_{2}^{\prime}\right), \\
\varphi=\left(\varphi_{0}, \varphi_{1}, \varphi_{2}\right), \quad \varphi^{\prime}=\left(\varphi_{0}^{\prime}, \varphi_{1}^{\prime}, \varphi_{2}^{\prime}\right) \in \mathcal{F}_{\mathrm{b}}^{(2)}\left(L_{2}\left(\mathbb{T}^{d}\right)\right) .
\end{gathered}
$$

Из определения операторов $\mathcal{A}_{m}, \mathcal{A}_{m}^{(s)}$ и $\Phi_{m}$ следует, что

$$
\Phi_{m} \mathcal{A}_{m} \Phi_{m}^{-1}=\operatorname{diag}\left\{\mathcal{A}_{m}^{(+)}, \mathcal{A}_{m}^{(-)}\right\}
$$

Учитывая унитарную эквивалентность операторов $\mathcal{A}_{m}$ и $\operatorname{diag}\left\{\mathcal{A}_{m}^{(+)}, \mathcal{A}_{m}^{(-)}\right\}$, получим искомую связь между спектрами операторов $\mathcal{A}_{m}$ и $\mathcal{A}_{m}^{(s)}$. Лемма доказана.

ЗАмечание 1 . Пусть $m=1,2$. Так как часть множества $\sigma_{\text {disc }}\left(\mathcal{A}_{m}^{(s)}\right)$ может лежать в множестве $\sigma_{\text {ess }}\left(\mathcal{A}_{m}^{(-s)}\right)$, имеют место соотношения

$$
\begin{aligned}
& \sigma_{\text {disc }}\left(\mathcal{A}_{m}\right) \subseteq \sigma_{\text {disc }}\left(\mathcal{A}_{m}^{(+)}\right) \cup \sigma_{\operatorname{disc}}\left(\mathcal{A}_{m}^{(-)}\right), \\
& \sigma_{\text {disc }}\left(\mathcal{A}_{m}\right)=\left\{\sigma_{\operatorname{disc}}\left(\mathcal{A}_{m}^{(+)}\right) \cup \sigma_{\operatorname{disc}}\left(\mathcal{A}_{m}^{(-)}\right)\right\} \backslash \sigma_{\mathrm{ess}}\left(\mathcal{A}_{m}\right) .
\end{aligned}
$$

Точнее,

$$
\sigma_{\mathrm{disc}}\left(\mathcal{A}_{m}\right)=\bigcup_{s= \pm}\left\{\sigma_{\mathrm{disc}}\left(\mathcal{A}_{m}^{(s)}\right) \backslash \sigma_{\mathrm{ess}}\left(\mathcal{A}_{m}^{(-s)}\right)\right\}
$$

Очевидно, что при $m=1,2$ и $s= \pm$ оператор $\mathcal{A}_{m}^{(s)}$ имеет более простую структуру, чем $\mathcal{A}_{m}$, и поэтому лемма 1 и соотношения $(2),(3)$ играют важную роль при дальнейших исследованиях спектра оператора $\mathcal{A}_{m}$. 


\section{3. НЕКОТОРЫЕ СПЕКТРАЛЬНЫЕ СВОЙСТВА ОПЕРАТОРА $\mathcal{A}_{1}$}

В этом разделе мы подробно изучим существенный и дискретный спектры оператора $\mathcal{A}_{1}^{(s)}, s= \pm$. При каждом $s= \pm$ рассмотрим оператор $\mathcal{A}_{1,0}^{(s)}$, действующий в $\mathcal{F}_{\mathrm{b}}^{(1)}\left(L_{2}\left(\mathbb{T}^{d}\right)\right)$ как

$$
\mathcal{A}_{1,0}^{(s)}:=\left(\begin{array}{cc}
0 & 0 \\
0 & \widehat{\mathcal{A}}_{11}^{(s)}
\end{array}\right) .
$$

Тогда оператор возмущения $\mathcal{A}_{1}^{(s)}-\mathcal{A}_{1,0}^{(s)}$ оператора $\mathcal{A}_{1,0}^{(s)}$ является самосопряженным оператором ранга 2. Из известной теоремы Вейля о сохранении существенного спектра при возмущениях конечного ранга следует, что существенный спектр оператора $\mathcal{A}_{1}^{(s)}$ совпадает с существенным спектром оператора $\mathcal{A}_{1,0}^{(s)}$. Известно, что

$$
\sigma_{\mathrm{ess}}\left(\mathcal{A}_{1,0}^{(s)}\right)=[-s \varepsilon,-s \varepsilon+M], \quad M:=\max _{p \in \mathbb{T}^{d}} w(p) .
$$

Отсюда вытекает, что $\sigma_{\text {ess }}\left(\mathcal{A}_{1}^{(s)}\right)=[-s \varepsilon,-s \varepsilon+M]$. Следовательно, в силу леммы 1 имеет место равенство

$$
\sigma_{\mathrm{ess}}\left(\mathcal{A}_{1}\right)=[-\varepsilon,-\varepsilon+M] \cup[\varepsilon, \varepsilon+M] .
$$

Напомним, что в непрерывном случае (см. статьи [2]-[5]) существенный спектр оператора $\mathcal{A}_{1}$ совпадает с полуосью $[-\varepsilon, \infty), \varepsilon>0$. В рассматриваемом случае существенный спектр оператора $\mathcal{A}_{1}$ есть объединение двух отрезков конечной длины, причем при $\varepsilon>M / 2$ они не пересекаются. Другими словами, при $\varepsilon>M / 2$ существенный спектр оператора $\mathcal{A}_{1}$ имеет лакуну $(-\varepsilon+M, \varepsilon)$.

Определим регулярную в $\mathbb{C} \backslash[-s \varepsilon,-s \varepsilon+M]$ функцию

$$
\Delta^{(s)}(z):=s \varepsilon-z-\alpha^{2} \int_{\mathbb{T}^{d}} \frac{v^{2}(t) d t}{-s \varepsilon+w(t)-z} .
$$

Функция $\Delta^{(s)}(\cdot)$ называется детерминантом Фредгольма, ассоциированным с оператором $\mathcal{A}_{1}^{(s)}$.

Следующая лемма устанавливает связь между собственными значениями оператора $\mathcal{A}_{1}^{(s)}$ и нулями функции $\Delta^{(s)}(\cdot)$.

Лемма 2. Число $z^{(s)} \in \mathbb{C} \backslash \sigma_{\mathrm{ess}}\left(\mathcal{A}_{1}^{(s)}\right)$ является собственным значением оператора $\mathcal{A}_{1}^{(s)}$ тогда и толъко тогда, когда $\Delta^{(s)}\left(z^{(s)}\right)=0$.

ДокаЗАтеЛьство. Пусть $z^{(s)} \in \mathbb{C} \backslash \sigma_{\mathrm{ess}}\left(\mathcal{A}_{1}^{(s)}\right)$ есть собственное значение оператора $\mathcal{A}_{1}^{(s)}$, и пусть $f=\left(f_{0}, f_{1}\right) \in \mathcal{F}_{\mathrm{b}}^{(1)}\left(L_{2}\left(\mathbb{T}^{d}\right)\right)$ - соответствующая собственная вектор-функция. Тогда эта вектор-функция удовлетворяет уравнению $\mathcal{A}_{1}^{(s)} f=z^{(s)} f$ или, что то же самое, системе уравнений

$$
\begin{array}{r}
\left(s \varepsilon-z^{(s)}\right) f_{0}+\alpha \int_{\mathbb{T}^{d}} v(t) f_{1}(t) d t=0, \\
\alpha v(p) f_{0}+\left(-s \varepsilon+w(p)-z^{(s)}\right) f_{1}(p)=0 .
\end{array}
$$

Так как $z^{(s)} \notin[-s \varepsilon,-s \varepsilon+M]$, из второго уравнения системы (4) для $f_{1}$ имеем

$$
f_{1}(p)=-\frac{\alpha v(p) f_{0}}{-s \varepsilon+w(p)-z^{(s)}} .
$$


Подставляя выражение (5) в первое уравнение системы (4), заключаем, что система (4) имеет ненулевое решение тогда и только тогда, когда $\Delta^{(s)}\left(z^{(s)}\right)=0$. Лемма доказана.

Из леммы 2 вытекает, что

$$
\sigma_{\text {disc }}\left(\mathcal{A}_{1}^{(s)}\right)=\left\{z \in \mathbb{C} \backslash \sigma_{\mathrm{ess}}\left(\mathcal{A}_{1}^{(s)}\right): \Delta^{(s)}(z)=0\right\} .
$$

Тогда, учитывая замечание 1, мы приходим к выводу, что

$$
\sigma_{\text {disc }}\left(\mathcal{A}_{1}\right)=\left\{z \in \mathbb{C} \backslash \sigma_{\text {ess }}\left(\mathcal{A}_{1}\right): \Delta^{(+)}(z) \Delta^{(-)}(z)=0\right\} .
$$

Обозначим через $I^{(m)}$ единичный оператор в $\mathcal{F}_{\mathrm{b}}^{(m)}\left(L_{2}\left(\mathbb{T}^{d}\right)\right), m=1,2$.

Лемма 3. Определитель $\Delta_{\mathcal{A}_{1}^{(s)} / \mathcal{A}_{1,0}^{(s)}}^{(s)}(z)$ возмущения оператора $\mathcal{A}_{1,0}^{(s)}$ относительно оператора $\mathcal{A}_{1}^{(s)}-\mathcal{A}_{1,0}^{(s)}$ имеет вид

$$
\Delta_{\mathcal{A}_{1}^{(s)} / \mathcal{A}_{1,0}^{(s)}}^{(s)}(z)=-\frac{1}{z} \Delta^{(s)}(z), \quad z \in \mathbb{C} \backslash \sigma\left(\mathcal{A}_{1,0}^{(s)}\right)
$$

ДокАЗАтельство. Так как $\mathcal{A}_{1}^{(s)}-\mathcal{A}_{1,0}^{(s)}$ является оператором ранга 2 , определитель возмущения $\Delta_{\mathcal{A}_{1}^{(s)} / \mathcal{A}_{1,0}^{(s)}}^{(s)}(z)$ может быть представлен выражением (см., например, работу [16])

$$
\Delta_{\mathcal{A}_{1}^{(s)} / \mathcal{A}_{1,0}^{(s)}}^{(s)}(z):=\operatorname{det}\left(I^{(1)}+\left(\mathcal{A}_{1}^{(s)}-\mathcal{A}_{1,0}^{(s)}\right)\left(\mathcal{A}_{1,0}^{(s)}-z\right)^{-1}\right) .
$$

Не нарушая общности, предположим, что $\|v\|=1$. Выберем ортонормированный базис $\left\{\psi_{n}\right\}_{n} \subset L_{2}\left(\mathbb{T}^{d}\right)$ следующим образом: $\psi_{1}:=v$ и $\psi_{j} \perp v$ для любых $j \geqslant 2$. Положим

$$
\Psi_{1}:=\frac{1}{\sqrt{2}}\left(\begin{array}{c}
1 \\
\psi_{1}
\end{array}\right), \quad \Psi_{2}:=\frac{1}{\sqrt{2}}\left(\begin{array}{c}
1 \\
-\psi_{1}
\end{array}\right), \quad \Psi_{j}:=\left(\begin{array}{c}
0 \\
\psi_{j-1}
\end{array}\right), \quad j \geqslant 3 .
$$

По построению система $\left\{\Psi_{n}\right\}_{n} \subset \mathcal{F}_{\mathrm{b}}^{(1)}\left(L_{2}\left(\mathbb{T}^{d}\right)\right)$ является ортонормированной. Пусть

$$
a_{i j}^{(s)}(z):=\left(\left(\mathcal{A}_{1}^{(s)}-\mathcal{A}_{1,0}^{(s)}\right)\left(\mathcal{A}_{1,0}^{(s)}-z\right)^{-1} \Psi_{i}, \Psi_{j}\right), \quad i, j \in \mathbb{N} .
$$

С помощью простых вычислений получим

$$
\begin{aligned}
& a_{11}^{(s)}(z)=-\frac{s \varepsilon}{z}+\alpha \int_{\mathbb{T}^{d}} \frac{v^{2}(t) d t}{-s \varepsilon+w(t)-z}-\frac{\alpha}{z}, \\
& a_{12}^{(s)}(z)=-\frac{s \varepsilon}{z}+\alpha \int_{\mathbb{T}^{d}} \frac{v^{2}(t) d t}{-s \varepsilon+w(t)-z}+\frac{\alpha}{z}, \\
& a_{21}^{(s)}(z)=-\frac{s \varepsilon}{z}-\alpha \int_{\mathbb{T}^{d}} \frac{v^{2}(t) d t}{-s \varepsilon+w(t)-z}-\frac{\alpha}{z}, \\
& a_{22}^{(s)}(z)=-\frac{s \varepsilon}{z}-\alpha \int_{\mathbb{T}^{d}} \frac{v^{2}(t) d t}{-s \varepsilon+w(t)-z}+\frac{\alpha}{z}, \\
& a_{i j}^{(s)}(z)=\delta_{i j} \quad \text { в остальных случаях, }
\end{aligned}
$$


где $\delta_{i j}-$ символ Кронекера. Следовательно,

$$
\Delta_{\mathcal{A}_{1}^{(s)} / \mathcal{A}_{1,0}^{(s)}}^{(s)}(z)=\frac{1}{4} \operatorname{det}\left(\begin{array}{cc}
2+a_{11}^{(s)}(z) & a_{12}^{(s)}(z) \\
a_{21}^{(s)}(z) & 2+a_{22}^{(s)}(z)
\end{array}\right)=-\frac{1}{z} \Delta^{(s)}(z) .
$$

Лемма доказана.

При дальнейшем исследовании собственных значений оператора $\mathcal{A}_{1}^{(s)}$ предположим, что

и положим

$$
\int_{\mathbb{T}^{d}} \frac{v^{2}(t) d t}{w(t)}<\infty, \quad \int_{\mathbb{T}^{d}} \frac{v^{2}(t) d t}{M-w(t)}<\infty
$$

$$
\begin{gathered}
\alpha_{1}:=\sqrt{M+2 \varepsilon}\left(\int_{\mathbb{T}^{d}} \frac{v^{2}(t) d t}{M-w(t)}\right)^{-1 / 2}, \quad \alpha_{2}:=\sqrt{2 \varepsilon}\left(\int_{\mathbb{T}^{d}} \frac{v^{2}(t) d t}{w(t)}\right)^{-1 / 2}, \\
\alpha_{3}:=\sqrt{M}\left(\int_{\mathbb{T}^{d}} \frac{v^{2}(t) d t}{2 \varepsilon+M-w(t)}\right)^{-1 / 2} .
\end{gathered}
$$

ЛЕмма 4. Имеют место следуюшие утверждения.

А. При всех $\alpha>0$ оператор $\mathcal{A}_{1}^{(-)}$имеет единственное простое собственное значение, лежащее левее $-\varepsilon$. Если $\alpha \in\left(0, \alpha_{1}\right]$, то оператор $\mathcal{A}_{1}^{(-)}$не имеет собственных значений, лежащих правее $M+\varepsilon$. При $\alpha>\alpha_{1}$ оператор $\mathcal{A}_{1}^{(-)}$имеет единственное простое собственное значение, лежащее правее $M+\varepsilon$.

Б. Если $\alpha \in\left(0, \min \left\{\alpha_{2}, \alpha_{3}\right\}\right]$, то оператор $\mathcal{A}_{1}^{(+)}$не имеет собственных значений, лежащих левее $-\varepsilon$ и правее $M+\varepsilon$. При $\alpha>\max \left\{\alpha_{2}, \alpha_{3}\right\}$ оператор $\mathcal{A}_{1}^{(+)}$имеет по одному простому собственному значению, лежащему левее - $\varepsilon$ и правее $M+\varepsilon$ соответственно.

ДокАЗАТЕЛЬство. Функция $\Delta^{(s)}(\cdot)$ строго убывает от $+\infty$ до $\Delta^{(s)}\left(z_{0}\right)$ в интервале $\left(-\infty, z_{0}\right), z_{0} \leqslant-\varepsilon$, и от $\Delta^{(s)}\left(z_{1}\right)$ до $-\infty$ в интервале $\left(z_{1},+\infty\right), z_{1} \geqslant M+\varepsilon$. Следовательно, оператор $\mathcal{A}_{1}^{(s)}$ имеет собственное значение $e_{0}<z_{0}$ тогда и только тогда, когда $\Delta^{(s)}\left(z_{0}\right)<0$, и имеет собственное значение $e_{1}>z_{1}$ тогда и только тогда, когда $\Delta^{(s)}\left(z_{1}\right)>0$.

А. Из соотношения

$$
\Delta^{(-)}(-\varepsilon)=-\alpha^{2} \int_{\mathbb{T}^{d}} \frac{v^{2}(t) d t}{w(t)+2 \varepsilon}<0
$$

следует, что при всех значениях параметра $\alpha>0$ оператор $\mathcal{A}_{1}^{(-)}$имеет одно простое собственное значение, лежащее левее $-\varepsilon$. Из определения числа $\alpha_{1}$ следуют формулы

$$
\begin{aligned}
& \Delta^{(-)}(M+\varepsilon)=-2 \varepsilon-M+\alpha^{2} \int_{\mathbb{T}^{d}} \frac{v^{2}(t) d t}{M-w(t)} \leqslant 0 \quad \Longleftrightarrow \alpha \in\left(0, \alpha_{1}\right], \\
& \Delta^{(-)}(M+\varepsilon)=-2 \varepsilon-M+\alpha^{2} \int_{\mathbb{T}^{d}} \frac{v^{2}(t) d t}{M-w(t)}>0 \quad \Longleftrightarrow \alpha>\alpha_{1},
\end{aligned}
$$

завершающие доказательство настоящего утверждения леммы. 
Б. Из определения чисел $\alpha_{2}, \alpha_{3}$ следует, что

$$
\begin{aligned}
\Delta^{(+)}(-\varepsilon)=2 \varepsilon-\alpha^{2} \int_{\mathbb{T}^{d}} \frac{v^{2}(t) d t}{w(t)} \geqslant 0 & \Longleftrightarrow \alpha \in\left(0, \alpha_{2}\right], \\
\Delta^{(+)}(-\varepsilon)=2 \varepsilon-\alpha^{2} \int_{\mathbb{T}^{d}} \frac{v^{2}(t) d t}{w(t)}<0 & \Longleftrightarrow \alpha \in\left(\alpha_{2},+\infty\right), \\
\Delta^{(+)}(M+\varepsilon)=-M+\alpha^{2} \int_{\mathbb{T}^{d}} \frac{v^{2}(t) d t}{2 \varepsilon+M-w(t)} \leqslant 0 & \Longleftrightarrow \alpha \in\left(0, \alpha_{3}\right], \\
\Delta^{(+)}(M+\varepsilon)=-M+\alpha^{2} \int_{\mathbb{T}^{d}} \frac{v^{2}(t) d t}{2 \varepsilon+M-w(t)}>0 & \Longleftrightarrow \alpha>\alpha_{3} .
\end{aligned}
$$

Следовательно, если $\alpha \in\left(0, \min \left\{\alpha_{2}, \alpha_{3}\right\}\right]$, то оператор $\mathcal{A}_{1}^{(+)}$не имеет собственных значений, лежащих левее $-\varepsilon$ и правее $M+\varepsilon$. При $\alpha>\max \left\{\alpha_{2}, \alpha_{3}\right\}$ оператор $\mathcal{A}_{1}^{(+)}$ имеет по одному простому собственному значению, лежащему левее $-\varepsilon$ и правее $M+\varepsilon$ соответственно. Лемма доказана.

Пусть

$$
\alpha_{\min }:=\min \left\{\alpha_{1}, \alpha_{2}, \alpha_{3}\right\}, \quad \alpha_{\max }:=\max \left\{\alpha_{1}, \alpha_{2}, \alpha_{3}\right\} .
$$

Следствием леммы 4 является следующее утверждение.

СлеДСТВИЕ 1. При всех $\alpha>0$ оператор $\mathcal{A}_{1}$ имеет не менее одного и не более четырех собственных значений. Более того, если $\alpha \in\left(0, \alpha_{\mathrm{min}}\right]$, то оператор $\mathcal{A}_{1}$ имеет единственное простое (изолированное) собственное значение, которое лежит левее $-\varepsilon$, а при $\alpha \in\left(\alpha_{\max },+\infty\right)$ оператор $\mathcal{A}_{1}$ имеет по два собственнъх значения, лежащих левее $-\varepsilon$ и правее $M+\varepsilon$ соответственно.

ЗАмечАниЕ 2. В следствии 1 собственное значение $E_{0}$ оператора $\mathcal{A}_{1}$, которое существует при всех $\alpha>0$, обычно называется основным состоянием. На основании доказательства леммы 2 можно сделать вывод, что компоненты соответствующей собственной вектор-функции выглядят следующим образом:

$$
f_{0}^{(+)}=0, \quad f_{0}^{(-)}=\mathrm{const} \neq 0, \quad f_{1}^{(+)}(p)=-\frac{\alpha v(p) f_{0}^{(-)}}{\varepsilon+w(p)-E_{0}}, \quad f_{1}^{(-)}(p)=0 .
$$

ЗАмЕчАНиЕ 3. На основании доказательства леммы 4 можно сделать вывод, что если интеграл

$$
\int_{\mathbb{T}^{d}} \frac{v^{2}(t) d t}{w(t)}
$$

расходится, то при всех $\alpha>0$ оператор $\mathcal{A}_{1}$ имеет два собственных значения, лежащих левее $-\varepsilon$. А при расходимости интеграла

$$
\int_{\mathbb{T}^{d}} \frac{v^{2}(t) d t}{M-w(t)}
$$

для любого $\alpha>0$ оператор $\mathcal{A}_{1}$ имеет по крайней мере одно собственное значение, лежащее правее $M+\varepsilon$. 
Верна следующая лемма.

Лемма 5. Пусть $\varepsilon>M / 2$. Тогда при всех $\alpha>0$ оператор $\mathcal{A}_{1}$ не имеет собственных значений, лежащих в лакуне $(M-\varepsilon, \varepsilon)$. Если $\alpha \in\left(0, \alpha_{3}\right)$, то оператор $\mathcal{A}_{1}$ имеет собственное значение, лежащее в существенном спектре, а именно в интервале $(\varepsilon, M+\varepsilon)$.

ДокАзАТЕЛЬство. Пусть $\varepsilon>M / 2$. В этом случае существенный спектр оператора $\mathcal{A}_{1}$ имеет лакуну $(M-\varepsilon, \varepsilon)$, причем при всех $\alpha>0$

$$
\begin{aligned}
\Delta^{(-)}(M-\varepsilon) & =-M-\alpha^{2} \int_{\mathbb{T}^{d}} \frac{v^{2}(t) d t}{2 \varepsilon+w(t)-M}<0, \\
\Delta^{(-)}(\varepsilon) & =-2 \varepsilon-\alpha^{2} \int_{\mathbb{T}^{d}} \frac{v^{2}(t) d t}{w(t)}<0, \\
\Delta^{(+)}(M-\varepsilon) & =2 \varepsilon-M+\alpha^{2} \int_{\mathbb{T}^{d}} \frac{v^{2}(t) d t}{M-w(t)}>0, \\
\Delta^{(+)}(\varepsilon) & =\alpha^{2} \int_{\mathbb{T}^{d}} \frac{v^{2}(t) d t}{2 \varepsilon-w(t)}>0 .
\end{aligned}
$$

Учитывая монотонность функции $\Delta^{(s)}(\cdot)$ в лакуне $(M-\varepsilon, \varepsilon)$ и лемму 2, получим, что оператор $\mathcal{A}_{1}$ не имеет собственных значений, лежащих в лакуне $(M-\varepsilon, \varepsilon)$. Очевидно, что при $\alpha \in\left(0, \alpha_{3}\right)$ имеет место равенство

$$
\Delta^{(+)}(M+\varepsilon)=-M+\alpha^{2} \int_{\mathbb{T}^{d}} \frac{v^{2}(t) d t}{2 \varepsilon+M-w(t)}<0 .
$$

Так как $\Delta^{(+)}(\cdot)$ является непрерывной монотонной функцией на полуоси $(M-\varepsilon,+\infty)$ и $\Delta^{(+)}(\varepsilon)>0$ (при этом $\varepsilon>M-\varepsilon$ ), то эта функция имеет простой нуль $z=E$ в лакуне $(\varepsilon, \varepsilon+M) \subset \sigma_{\text {ess }}\left(\mathcal{A}_{1}\right)$. Нетрудно убедиться, что вектор-функция

$$
\begin{gathered}
F=\left\{f_{0}^{(\sigma)}, f_{1}^{(\sigma)}, \sigma= \pm\right\}, \\
f_{0}^{(+)}=\mathrm{const} \neq 0, \quad f_{0}^{(-)}=0, \quad f_{1}^{(+)}(p)=0, \quad f_{1}^{(-)}(p)=-\frac{\alpha v(p) f_{0}^{(+)}}{-\varepsilon+w(p)-E},
\end{gathered}
$$

удовлетворяет уравнению $\mathcal{A}_{1} F=E F$ и $F \in \mathcal{L}_{1}$, т. е. число $z=E$ является собственным значением оператора $\mathcal{A}_{1}$. Лемма доказана.

Из рассуждений, приведенных в этом разделе, можно сделать вывод, что существование изолированных или вложенных собственных значений оператора $\mathcal{A}_{1}$ тесно связано со свойствами операторов $\mathcal{A}_{1}^{(s)}, s= \pm$, и $\sigma_{\operatorname{disc}}\left(\mathcal{A}_{1}\right) \neq \varnothing$. Полученные ранее в работах [11], [13]-[15] результаты для $\mathcal{A}_{1}^{(s)}, s= \pm$, недостаточны для данного вывода.

\section{4. УРАВНЕНИЕ ФАДДЕЕВА ДЛЯ СОБСТВЕННЫХ ВЕКТОР-ФУНКЦИЙ ОПЕРАТОРА $\mathcal{A}_{2}^{(s)}$}

В этом разделе получен аналог системы интегральных уравнений Фаддеева и его симметризованный вариант для собственных вектор-функций оператора $\mathcal{A}_{2}^{(s)}$, играющий важную роль при исследовании спектра этого оператора. 
Напомним, что для $\lambda \in \mathbb{R}$ и $A \subset \mathbb{R}$ имеет место равенство $\lambda+A=\{\lambda+a: a \in A\}$. Обозначим

$$
\sigma^{(s)}:=\bigcup_{p \in \mathbb{T}^{d}}\left\{w(p)+\sigma_{\operatorname{disc}}\left(\mathcal{A}_{1}^{(-s)}\right)\right\}, \quad \Sigma^{(s)}:=\sigma^{(s)} \cup[s \varepsilon, s \varepsilon+2 M] .
$$

Следует отметить, что

$$
\bigcup_{p \in \mathbb{T}^{d}}\left\{w(p)+\sigma_{\mathrm{ess}}\left(\mathcal{A}_{1}^{(-s)}\right)\right\}=[s \varepsilon, s \varepsilon+2 M]
$$

поэтому имеет место равенство

$$
\bigcup_{p \in \mathbb{T}^{d}}\left\{w(p)+\sigma\left(\mathcal{A}_{1}^{(-s)}\right)\right\}=\Sigma^{(s)} .
$$

При каждом $z \in \mathbb{C} \backslash \Sigma^{(s)}$ определим блочно-операторную матрицу $T^{(s)}(z)$, действующую в пространстве $\mathcal{F}_{\mathrm{b}}^{(1)}\left(L_{2}\left(\mathbb{T}^{d}\right)\right)$, следующим образом:

$$
T^{(s)}(z):=\left(\begin{array}{cc}
T_{00}^{(s)}(z) & T_{01}^{(s)}(z) \\
T_{10}^{(s)}(z) & T_{11}^{(s)}(z)
\end{array}\right)
$$

где матричные элементы суть

$$
\begin{aligned}
& T_{00}^{(s)}(z) g_{0}=(1+z-s \varepsilon) g_{0}, \quad T_{01}^{(s)}(z) g_{1}=-\alpha \int_{\mathbb{T}^{d}} v(t) g_{1}(t) d t \\
& \left(T_{10}^{(s)}(z) g_{0}\right)(p)=-\frac{\alpha v(p) g_{0}}{\Delta^{(-s)}(z-w(p))}, \\
& \left(T_{11}^{(s)}(z) g_{1}\right)(p)=\frac{\alpha^{2} v(p)}{\Delta^{(-s)}(z-w(p))} \int_{\mathbb{T}^{d}} \frac{v(t) g_{1}(t) d t}{s \varepsilon+w(p)+w(t)-z}, \\
& \quad\left(g_{0}, g_{1}\right) \in \mathcal{F}_{\mathrm{b}}^{(1)}\left(L_{2}\left(\mathbb{T}^{d}\right)\right) .
\end{aligned}
$$

Установим связь между собственными значениями операторов $\mathcal{A}_{2}^{(s)}$ и $T^{(s)}(z)$.

ЛЕмма 6. Число $z \in \mathbb{C} \backslash \Sigma^{(s)}$ является собственнъм значением оператора $\mathcal{A}_{2}^{(s)}$ тогда и только тогда, когда оператор $T^{(s)}(z)$ имеет собственное значение, равное единище, причем их кратности совпадают.

ДоказАтельство. Пусть $z \in \mathbb{C} \backslash \Sigma^{(s)}$ - собственное значение оператора $\mathcal{A}_{2}^{(s)}$ и $f=\left(f_{0}, f_{1}, f_{2}\right) \in \mathcal{F}_{\mathrm{b}}^{(2)}\left(L_{2}\left(\mathbb{T}^{d}\right)\right)$ - соответствующая собственная вектор-функция. Тогда элементы $f_{0}, f_{1}$ и $f_{2}$ удовлетворяют системе уравнений

$$
\begin{array}{r}
(s \varepsilon-z) f_{0}+\alpha \int_{\mathbb{T}^{d}} v(t) f_{1}(t) d t=0, \\
\alpha v(p) f_{0}+(-s \varepsilon+w(p)-z) f_{1}(p)+\alpha \int_{\mathbb{T}^{d}} v(t) f_{2}(p, t) d t=0, \\
\alpha\left(v(p) f_{1}(q)+v(q) f_{1}(p)\right)+(s \varepsilon+w(p)+w(q)-z) f_{2}(p, q)=0 .
\end{array}
$$

Так как $z \notin[s \varepsilon, s \varepsilon+2 M]$, из третьего уравнения системы (7) получим равенство

$$
f_{2}(p, q)=-\frac{\alpha\left(v(q) f_{1}(p)+v(p) f_{1}(q)\right)}{s \varepsilon+w(p)+w(q)-z} .
$$


Подставляя выражение (8) во второе уравнение системы (7) получим, что система уравнений

$$
\begin{gathered}
0=(z-s \varepsilon) f_{0}-\alpha \int_{\mathbb{T}^{d}} v(t) f_{1}(t) d t \\
\Delta^{(-s)}(z-w(p)) f_{1}(p)=-\alpha v(p) f_{0}+\alpha^{2} v(p) \int_{\mathbb{T}^{d}} \frac{v(t) f_{1}(t) d t}{s \varepsilon+w(p)+w(t)-z}
\end{gathered}
$$

имеет нетривиальное решение тогда и только тогда, когда система уравнений (7) имеет нетривиальное решение и линейные подпространства, порожденные решениями систем (7) и (9), имеют одинаковые размерности.

По определению множества $\sigma^{(s)}$ для любых $z \notin \sigma^{(s)}$ и $p \in \mathbb{T}^{d}$ имеет место неравенство $\Delta^{(-s)}(z-w(p)) \neq 0$. Следовательно, система уравнений (9) имеет нетривиальное решение тогда и только тогда, когда система уравнений

$$
\begin{aligned}
f_{0} & =(1+z-s \varepsilon) f_{0}-\alpha \int_{\mathbb{T}^{d}} v(t) f_{1}(t) d t \\
f_{1}(p) & =-\frac{\alpha v(p) f_{0}}{\Delta^{(-s)}(z-w(p))}+\frac{\alpha^{2} v(p)}{\Delta^{(-s)}(z-w(p))} \int_{\mathbb{T}^{d}} \frac{v(t) f_{1}(t) d t}{s \varepsilon+w(p)+w(t)-z}
\end{aligned}
$$

или матричное уравнение

$$
g=T^{(s)}(z) g, \quad g=\left(g_{0}, g_{1}\right) \in \mathcal{F}_{\mathrm{b}}^{(1)}\left(L_{2}\left(\mathbb{T}^{d}\right)\right),
$$

имеют нетривиальные решения. Здесь вновь линейные подпространства, порожденные решениями системы уравнений (10) и уравнения (11), имеют одинаковые размерности. Лемма доказана.

Операторное уравнение (11) обычно называется аналогом уравнения Фаддеева для собственных функций оператора $\mathcal{A}_{2}^{(s)}$.

По определению множества $\sigma^{(s)}$ для любого $(a, b) \subset \mathbb{R} \backslash \Sigma^{(s)}$ функция $\Delta^{(s)}(z-w(p))$ не меняет знак на интервале $(a, b)$ независимо от значений $p \in \mathbb{T}^{d}$. При каждом фиксированном $z \in \mathbb{R} \backslash \Sigma^{(s)}$ определим $\xi_{z}^{(s)}$ равенством: $\xi_{z}^{(s)}:=\operatorname{sgn}\left(\Delta^{(-s)}(z-w(p))\right)$. $\mathrm{B}$ частности, $\xi_{z}^{(s)}=+$ для любого $z<\min \Sigma^{(s)}$ и $\xi_{z}^{(s)}=-$ для любого $z>\max \Sigma^{(s)}$. При каждом $z \in \mathbb{R} \backslash \Sigma^{(s)}$ определим блочно-операторную матрицу $\widehat{T}^{(s)}(z)$, действующую в пространстве $\mathcal{F}_{\mathrm{b}}^{(1)}\left(L_{2}\left(\mathbb{T}^{d}\right)\right)$, следующим образом:

$$
\widehat{T}^{(s)}(z):=\left(\begin{array}{cc}
\widehat{T}_{00}^{(s)}(z) & \widehat{T}_{01}^{(s)}(z) \\
\widehat{T}_{10}^{(s)}(z) & \widehat{T}_{11}^{(s)}(z)
\end{array}\right),
$$

где матричные элементы суть

$$
\begin{gathered}
\widehat{T}_{00}^{(s)}(z) g_{0}=\left(1+\xi_{z}^{(s)}(z-s \varepsilon)\right) g_{0}, \quad T_{01}^{(s)}(z) g_{1}=-\xi_{z}^{(s)} \alpha \int_{\mathbb{T}^{d}} \frac{v(t) g_{1}(t) d t}{\sqrt{\left|\Delta^{(-s)}(z-w(t))\right|}}, \\
\left(\widehat{T}_{10}^{(s)}(z) g_{0}\right)(p)=-\xi_{z}^{(s)} \frac{\alpha v(p) g_{0}}{\sqrt{\left|\Delta^{(-s)}(z-w(p))\right|}}, \\
\left(\widehat{T}_{11}^{(s)}(z) g_{1}\right)(p)=\xi_{z}^{(s)} \frac{\alpha^{2} v(p)}{\sqrt{\left|\Delta^{(-s)}(z-w(p))\right|}} \int_{\mathbb{T}^{d}} \frac{v(t) g_{1}(t) d t}{\sqrt{\left|\Delta^{(-s)}(z-w(t))\right|}(s \varepsilon+w(p)+w(t)-z)}, \\
\left(g_{0}, g_{1}\right) \in \mathcal{F}_{\mathrm{b}}^{(1)}\left(L_{2}\left(\mathbb{T}^{d}\right)\right) .
\end{gathered}
$$


Лемма 7. Пусть $z \in \mathbb{R} \backslash \Sigma^{(s)}$. Число $\lambda=1$ является собственным значением оператора $T^{(s)}(z)$ тогда и толъко тогда, когда это число также является собственным значением оператора $\widehat{T}^{(s)}(z)$, причем их кратности совпадают.

ДокАЗАТЕЛьство. Пусть число $\lambda=1$ является собственным значением оператора $T^{(s)}(z)$. Тогда система уравнений $(10)$ или же эквивалентная ей система уравнений $(9)$ имеют нетривиальное решение. По определению множества $\sigma^{(s)}$ и числа $\xi_{z}^{(s)}$ имеем $\xi_{z}^{(s)} \Delta^{-(s)}(z-w(p))>0$ при всех значениях $p \in \mathbb{T}^{d}$ и $z \in \mathbb{R} \backslash \Sigma^{(s)}$. Умножая оба уравнения системы $(9)$ на $\xi_{z}^{(s)}$, получим, что система уравнений

$$
\begin{aligned}
g_{0}= & \left(1+\xi_{z}^{(s)}(z-s \varepsilon)\right) g_{0}-\xi_{z}^{(s)} \alpha \int_{\mathbb{T}^{d}} \frac{v(t) g_{1}(t) d t}{\sqrt{\left|\Delta^{(-s)}(z-w(t))\right|}}, \\
g_{1}(p)= & -\xi_{z}^{(s)} \frac{\alpha v(p) g_{0}}{\sqrt{\left|\Delta^{(-s)}(z-w(p))\right|}}+ \\
& +\xi_{z}^{(s)} \frac{\alpha^{2} v(p)}{\sqrt{\left|\Delta^{(-s)}(z-w(p))\right|}} \int_{\mathbb{T}^{d}} \frac{v(t) g_{1}(t) d t}{\sqrt{\left|\Delta^{(-s)}(z-w(t))\right|}(s \varepsilon+w(p)+w(t)-z)}
\end{aligned}
$$

или матричное уравнение

$$
g=\widehat{T}^{(s)}(z) g, \quad g=\left(g_{0}, g_{1}\right) \in \mathcal{F}_{\mathrm{b}}^{(1)}\left(L_{2}\left(\mathbb{T}^{d}\right)\right)
$$

имеют нетривиальное решение, причем линейные подпространства, порожденные решениями системы (12) и уравнения (13), имеют одинаковые размерности. Лемма доказана.

Операторное уравнение (13) обычно называется симметризованным вариантом уравнения Фаддеева для собственных вектор-функций оператора $\mathcal{A}_{2}^{(s)}$. Симметризованный компактный оператор $\widehat{T}^{(s)}(z)$ играет важную роль при исследовании дискретного спектра оператора $\mathcal{A}_{2}^{(s)}$.

\section{5. СУЩЕСТВЕННЫЙ СПЕКТР ОПЕРАТОРА $\mathcal{A}_{2}$}

В настоящем разделе изучены местоположение и структура существенного спектра оператора $\mathcal{A}_{2}$. Получена оценка для нижней грани существенного спектра опеpaтора $\mathcal{A}_{2}$.

Напомним, что норма элемента $f=\left(f_{0}, f_{1}, f_{2}\right) \in \mathcal{F}_{\mathrm{b}}^{(2)}\left(L_{2}\left(\mathbb{T}^{d}\right)\right)$ определяется выражением

$$
\|f\|^{2}:=\left|f_{0}\right|^{2}+\int_{\mathbb{T}^{d}}\left|f_{1}(p)\right|^{2} d p+\frac{1}{2} \int_{\left(\mathbb{T}^{d}\right)^{2}}\left|f_{2}(p, q)\right|^{2} d p d q .
$$

Следующая теорема описывает местоположение существенного спектра оператоpa $\mathcal{A}_{2}$.

Teорема 1. Существенный спектр $\sigma_{\mathrm{ess}}\left(\mathcal{A}_{2}\right)$ оператора $\mathcal{A}_{2}$ совпадает со множеством $\Sigma^{(+)} \cup \Sigma^{(-)}$, т.е. справедливо равенство $\sigma_{\mathrm{ess}}\left(\mathcal{A}_{2}\right)=\Sigma^{(+)} \cup \Sigma^{(-)}$. Более того, множество $\sigma_{\mathrm{ess}}\left(\mathcal{A}_{2}\right)$ представляет собой обгединение не более чем шести отрезков.

ДокАзАТЕльство. В силу леммы 1 справедливо следующее выражение для существенного спектра оператора $\mathcal{A}_{2}: \sigma_{\mathrm{ess}}\left(\mathcal{A}_{2}\right)=\sigma_{\mathrm{ess}}\left(\mathcal{A}_{2}^{(+)}\right) \cup \sigma_{\mathrm{ess}}\left(\mathcal{A}_{2}^{(-)}\right)$. Покажем, что $\sigma_{\mathrm{ess}}\left(\mathcal{A}_{2}^{(s)}\right)=\Sigma^{(s)}$. По определению $\Sigma^{(s)}=\sigma^{(s)} \cup[s \varepsilon, s \varepsilon+2 M]$.

5 Теоретическая и математическая физика, т. 186, № 2, 2016 г. 
Докажем, что $[s \varepsilon, s \varepsilon+2 M] \subset \sigma_{\text {ess }}\left(\mathcal{A}_{2}^{(s)}\right)$. Пусть $z_{0} \in[s \varepsilon, s \varepsilon+2 M]-$ произвольная точка. Покажем, что $z_{0} \in \sigma_{\mathrm{ess}}\left(\mathcal{A}_{2}^{(s)}\right)$. Для этого удобно воспользоваться критерием Вейля [17], т.е. достаточно построить последовательность ортонормированных векторов $\left\{f^{(n)}\right\} \subset \mathcal{F}_{\mathrm{b}}^{(2)}\left(L_{2}\left(\mathbb{T}^{d}\right)\right)$, для которых $\left\|\left(\mathcal{A}_{2}^{(s)}-z_{0} I^{(2)}\right) f^{(n)}\right\| \rightarrow 0$ при $n \rightarrow \infty$. Здесь символ $\|\cdot\|$ означает норму в $\mathcal{F}_{\mathrm{b}}^{(2)}\left(L_{2}\left(\mathbb{T}^{d}\right)\right)$, определенную равенством (14). Так как $w(\cdot)$ - непрерывная функция в компактном множестве $\mathbb{T}^{d}$, существует точка $\left(p_{0}, q_{0}\right) \in\left(\mathbb{T}^{d}\right)^{2}$ такая, что $z_{0}=s \varepsilon+w\left(p_{0}\right)+w\left(q_{0}\right)$. При $n \in \mathbb{N}$ рассмотрим следующую выколотую окрестность точки $p_{0} \in \mathbb{T}^{d}$ :

$$
V_{n}\left(p_{0}\right):=\left\{p \in \mathbb{T}^{d}: \frac{1}{n+1}<\left|p-p_{0}\right|<\frac{1}{n}\right\}
$$

где

$$
|p|:=\sqrt{p_{1}^{2}+\cdots+p_{d}^{2}}, \quad p=\left(p_{1}, \ldots, p_{d}\right) \in \mathbb{T}^{d} .
$$

Пусть $\mu(\Omega)$ - мера Лебега множества $\Omega$ и $\chi_{\Omega}(\cdot)$ - характеристическая функция множества $\Omega$. Если $p_{0} \neq q_{0}$, то при $n_{0}:=\left[2 /\left|p_{0}-q_{0}\right|\right]+1$ верно следующее равенство: $V_{n_{0}}\left(p_{0}\right) \cap V_{n_{0}}\left(q_{0}\right)=\varnothing$. Тогда оно верно и при всех $n \geqslant n_{0}$. Выберем последовательность вектор-функций $\left\{f^{(n)}\right\}_{n \geqslant n_{0}} \subset \mathcal{F}_{\mathrm{b}}^{(2)}\left(L_{2}\left(\mathbb{T}^{d}\right)\right)$ следующим образом:

$$
f^{(n)}:=\left(\begin{array}{c}
0 \\
0 \\
f_{2}^{(n)}
\end{array}\right), \quad f_{2}^{(n)}(p, q):=\frac{1}{\mu\left(V_{n}\left(p_{0}\right)\right)} \chi_{W_{n}}(p, q), \quad n \geqslant n_{0},
$$

где $W_{n}:=V_{n}\left(p_{0}\right) \times V_{n}\left(q_{0}\right) \cup V_{n}\left(q_{0}\right) \times V_{n}\left(p_{0}\right)$. Очевидно, что $\left\{f^{(n)}\right\}-$ ортонормированная последовательность. При каждом значении номера $n \geqslant n_{0}$ рассмотрим функцию $\left(\mathcal{A}_{2}^{(s)}-z_{0} I^{(2)}\right) f^{(n)}$ и оценим ее норму:

$$
\begin{aligned}
\left\|\left(\mathcal{A}_{2}^{(s)}-z_{0} I^{(2)}\right) f^{(n)}\right\|^{2} \leqslant & 4 \alpha^{2} \mu\left(V_{n}\left(p_{0}\right)\right) \max _{p \in \mathbb{T}^{d}}|v(p)|^{2}+ \\
& +\sup _{(p, q) \in V_{n}\left(p_{0}\right) \times V_{n}\left(q_{0}\right)}\left|s \varepsilon+w(p)+w(q)-z_{0}\right|^{2} .
\end{aligned}
$$

Таким образом, из определения множества $V_{n}\left(p_{0}\right)$ и из непрерывности функции $w(\cdot)$ следует, что $\left\|\left(\mathcal{A}_{2}^{(s)}-z_{0} I^{(2)}\right) f^{(n)}\right\| \rightarrow 0$ при $n \rightarrow \infty$, т. е. $z_{0} \in \sigma_{\mathrm{ess}}\left(\mathcal{A}_{2}^{(s)}\right)$. В силу произвольности точки $z_{0}$ верно включение $[s \varepsilon, s \varepsilon+2 M] \subset \sigma_{\mathrm{ess}}\left(\mathcal{A}_{2}^{(s)}\right)$.

При $p_{0}=q_{0}$ выберем последовательность функций $f_{2}^{(n)}, n \in \mathbb{N}$, как

$$
f_{2}^{(n)}(p, q):=\frac{\sqrt{2}}{\mu\left(V_{n}\left(p_{0}\right)\right)} \chi_{V_{n}\left(p_{0}\right)}(p) \chi_{V_{n}\left(p_{0}\right)}(q)
$$

и получим, что верно включение $[s \varepsilon, s \varepsilon+2 M] \subset \sigma_{\mathrm{ess}}\left(\mathcal{A}_{2}^{(s)}\right)$.

Теперь докажем, что $\sigma^{(s)} \subset \sigma_{\mathrm{ess}}\left(\mathcal{A}_{2}^{(s)}\right)$. Пусть $z_{1} \in \sigma^{(s)}$ - произвольная точка. Покажем, что $z_{1} \in \sigma_{\mathrm{ess}}\left(\mathcal{A}_{2}^{(s)}\right)$. Для этого вновь построим последовательность ортонормированных вектор-функций $f^{(n)}$ такую, что $\left\|\left(\mathcal{A}_{2}^{(s)}-z_{1} I^{(2)}\right) f^{(n)}\right\| \rightarrow 0$ при $n \rightarrow \infty$.

Далее возможны два случая: $z_{1} \in[s \varepsilon, s \varepsilon+2 M]$ и $z_{1} \notin[s \varepsilon, s \varepsilon+2 M]$. Если $z_{1} \in$ $[s \varepsilon, s \varepsilon+2 M]$, то, как показано выше, $z_{1} \in \sigma_{\text {ess }}\left(\mathcal{A}_{2}^{(s)}\right)$. Если $z_{1} \notin[s \varepsilon, s \varepsilon+2 M]$, то по определению множества $\sigma^{(s)}$ существуют точки $p_{1} \in \mathbb{T}^{d}$ и $\lambda_{1} \in \sigma_{\operatorname{disc}}\left(\mathcal{A}_{1}^{(-s)}\right)$ такие, 
что $z_{1}=w\left(p_{1}\right)+\lambda_{1}$. Таким образом, существует такая единичная вектор-функция $\Psi=\left(\psi_{0}, \psi_{1}\right) \in \mathcal{F}_{\mathrm{b}}^{(1)}\left(L_{2}\left(\mathbb{T}^{d}\right)\right)$, что

$$
\left(\mathcal{A}_{1}^{(-s)}-\lambda_{1} I^{(1)}\right) \Psi=0 .
$$

Положим

$$
\tilde{f}^{(n)}:=\left(\begin{array}{c}
0 \\
f_{1}^{(n)} \\
f_{2}^{(n)}
\end{array}\right), \quad \begin{aligned}
f_{1}^{(n)}(p) & :=\frac{c_{n}(p) \psi_{0}}{\sqrt{\mu\left(V_{n}\left(p_{1}\right)\right)}} \\
f_{2}^{(n)}(p, q) & :=\frac{1}{\sqrt{\mu\left(V_{n}\left(p_{1}\right)\right)}}\left(c_{n}(q) \psi_{1}(p)+c_{n}(p) \psi_{1}(q)\right) .
\end{aligned}
$$

Здесь функции $\left\{c_{n}\right\} \subset L_{2}\left(\mathbb{T}^{d}\right)$ образуют ортонормированную систему, удовлетворяющую условию $\operatorname{supp}\left(c_{n}\right) \subset V_{n}\left(p_{1}\right), n \in \mathbb{N}$, которую можно определить из условия ортогональности функций $\left\{\tilde{f}^{(n)}\right\}$ : при $n \neq k$

$$
\left(\tilde{f}^{(n)}, \tilde{f}^{(k)}\right)=\frac{1}{2 \sqrt{\mu\left(V_{n}\left(p_{1}\right)\right) \mu\left(V_{k}\left(p_{1}\right)\right)}} \int_{V_{n}\left(p_{1}\right)} c_{n}(p) \overline{\psi_{1}(p)} d p \int_{V_{k}\left(p_{1}\right)} \overline{c_{k}(q)} \psi_{1}(q) d q=0 .
$$

Это означает, что $\int_{V_{n}\left(p_{1}\right)} c_{n}(p) \overline{\psi_{1}(p)} d p=0$ для любого $n \in \mathbb{N}$. При этом существование функций $c_{n}(\cdot)$ вытекает из бесконечномерности пространства $L_{2}\left(V_{n}\left(p_{1}\right)\right)$.

Прямые вычисления показывают, что

$$
\left\|\tilde{f}^{(n)}\right\|=\frac{1}{\sqrt{\mu\left(V_{n}\left(p_{1}\right)\right)}}>0
$$

Положим $f^{(n)}:=\tilde{f}^{(n)} /\left\|\tilde{f}^{(n)}\right\|$. Очевидно, что система функций $\left\{f^{(n)}\right\}$ является ортонормированной. Покажем, что для этой системы при $z_{1} \in \sigma^{(s)}$ верно равенство

$$
\lim _{n \rightarrow+\infty}\left\|\left(\mathcal{A}_{2}^{(s)}-z_{1} I^{(2)}\right) f^{(n)}\right\|=0 .
$$

Заметим, что

$$
\left\|\left(\mathcal{A}_{2}^{(s)}-z_{1} I^{(2)}\right) f^{(n)}\right\|^{2} \leqslant 2 \sup _{p \in V_{n}\left(p_{1}\right)}\left|w(p)-w\left(p_{1}\right)\right|^{2}+\alpha^{2} \mu\left(V_{n}\left(p_{1}\right)\right) \max _{p \in \mathbb{T}^{d}}|v(p)|^{2} .
$$

Тогда в силу непрерывности функции $w(\cdot)$ на $\mathbb{T}^{d}$ и по построению множества $V_{n}\left(p_{1}\right)$ имеем $\left\|\left(\mathcal{A}_{2}^{(s)}-z_{1} I^{(2)}\right) f^{(n)}\right\| \rightarrow 0$ при $n \rightarrow \infty$. Таким образом, $z_{1} \in \sigma_{\text {ess }}\left(\mathcal{A}_{2}^{(s)}\right)$. Поскольку точка $z_{1} \in \sigma^{(s)}$ произвольна, верно включение $\sigma^{(s)} \subset \sigma_{\text {ess }}\left(\mathcal{A}_{2}^{(s)}\right)$.

Теперь докажем обратное включение, а именно $\sigma_{\mathrm{ess}}\left(\mathcal{A}_{2}^{(s)}\right) \subset \Sigma^{(s)}$. По построению $T^{(s)}(z)$ является компактнозначной аналитической функцией на $\mathbb{C} \backslash \Sigma^{(s)}$. Из самосопряженности оператора $\mathcal{A}_{2}^{(s)}$ и леммы 6 следует, что операторнозначная функция $\left(I^{(1)}-T^{(s)}(z)\right)^{-1}$ существует при $\operatorname{Im}(z) \neq 0$. Согласно аналитической теореме Фредгольма (см. теорему ХІІІ.13 из монографии [17]) операторнозначная функция $\left(I^{(1)}-T^{(s)}(z)\right)^{-1}$ существует на $\mathbb{C} \backslash \Sigma^{(s)}$ всюду за исключением дискретного множества $\mathcal{M}$, где она имеет вычеты конечного ранга. Это означает, что множество $\sigma\left(\mathcal{A}_{2}^{(s)}\right) \backslash \Sigma^{(s)}$ состоит только из изолированных точек, которые могут иметь предельные точки только в граничных точках множества $\Sigma^{(s)}$. Отсюда следует, что $\sigma\left(\mathcal{A}_{2}^{(s)}\right) \backslash \Sigma^{(s)} \subset \sigma\left(\mathcal{A}_{2}^{(s)}\right) \backslash \sigma_{\text {ess }}\left(\mathcal{A}_{2}^{(s)}\right)$, т. е. справедливо включение $\sigma_{\text {ess }}\left(\mathcal{A}_{2}^{(s)}\right) \subset \Sigma^{(s)}$. Таким образом, мы доказали, что $\sigma_{\mathrm{ess}}\left(\mathcal{A}_{2}^{(s)}\right)=\Sigma^{(s)}$. 
Осталось доказать, что множество $\sigma_{\mathrm{ess}}\left(\mathcal{A}_{2}^{(s)}\right)$ представляет собой объединение не более чем трех отрезков. В силу леммы 4 оператор $\mathcal{A}_{1}^{(s)}$ имеет не более двух простых собственных значений, лежащих вне отрезка $[s \varepsilon, s \varepsilon+2 M]$. Так как функция $w(\cdot)$ непрерывна на множестве $\mathbb{T}^{d}$, множество $\sigma^{(s)}=\cup_{p \in \mathbb{T}^{d}}\left\{w(p)+\sigma_{\text {disc }}\left(\mathcal{A}_{1}^{(-s)}\right)\right\}$ представляет собой объединение не более чем двух отрезков. Следовательно, множество $\sigma_{\text {ess }}\left(\mathcal{A}_{2}^{(s)}\right)$ есть объединение не более чем трех отрезков. Применив лемму 1 , завершим доказательство теоремы.

Введем новые подмножества существенного спектра оператора $\mathcal{A}_{2}$.

ОПРЕДЕЛЕНИЕ 1. Множества

$$
\sigma_{\text {two }}\left(\mathcal{A}_{2}\right):=\sigma^{(+)} \cup \sigma^{(-)}, \quad \sigma_{\text {three }}\left(\mathcal{A}_{2}\right):=[-\varepsilon,-\varepsilon+2 M] \cup[\varepsilon, \varepsilon+2 M]
$$

называются двухчастичной и трехчастичной ветвями существенного спектра оператора $\mathcal{A}_{2}$ соответственно.

Из определения множества $\sigma_{\text {three }}\left(\mathcal{A}_{2}\right)$ следует, что $\min \left(\sigma_{\text {three }}\left(\mathcal{A}_{2}\right)\right)=-\varepsilon$. Заметим, что в работе [13] существенный спектр оператора $\mathcal{A}_{2}^{(s)}$ в более общем виде описан в терминах нулей определителя Фредгольма $\Delta^{(-s)}(z-w(p))$ и спектра оператора $A_{22}^{(s)}$. По определению множество $\sigma^{(s)}$ состоит из таких точек $z$ множества $\mathbb{C} \backslash[s \varepsilon+w(p), s \varepsilon+M+w(p)]$, для которых $\Delta^{(-s)}(z-w(p))=0$ при некотором $p \in \mathbb{T}^{d}$. Для формулировки следующего результата введем обозначения

$$
\begin{gathered}
\sigma_{1}^{(s)}:=\sigma^{(s)} \cap(-\infty, s \varepsilon], \quad \sigma_{2}^{(s)}:=\sigma^{(s)} \cap[s \varepsilon+2 M, \infty), \\
E_{\min }^{(k, s)}:=\min \left(\sigma_{k}^{(s)}\right), \quad E_{\max }^{(k, s)}:=\min \left(\sigma_{k}^{(s)}\right), \quad k=1,2, \\
E_{\min }:=\min \left\{E_{\min }^{(1,+)}, E_{\min }^{(1,-)}\right\} .
\end{gathered}
$$

В случае $\sigma_{k}^{(s)}=\varnothing$ при $k=1,2$ положим

$$
E_{\min }^{(1, s)}=E_{\max }^{(1, s)}=s \varepsilon, \quad E_{\min }^{(2, s)}=E_{\max }^{(2, s)}=s \varepsilon+2 M .
$$

Следующая теорема описывает структуру существенного спектра оператора $\mathcal{A}_{2}$ в зависимости от значений параметра взаимодействия $\alpha>0$.

Теорема 2. Для существенного спектра оператора $\mathcal{A}_{2}$ верны следующие утверждения.

А. Для любого $\alpha>0$ справедлива оченка $E_{\min }<-\varepsilon$.

Б. Пусть выполняется условие (6). Тогда мы имеем следующее.

Б1. Если $\alpha \in\left(0, \alpha_{\min }\right]$, mo

$$
\sigma_{\mathrm{ess}}\left(\mathcal{A}_{2}\right)=\left[E_{\min }^{(1,-)}, E_{\max }^{(1,-)}\right] \cup[-\varepsilon,-\varepsilon+2 M] \cup[\varepsilon, \varepsilon+2 M],
$$

где $E_{\min }^{(1,-)}<-\varepsilon$.

Б2. Если $\alpha \in\left(\alpha_{1}, \infty\right)$, то

$\sigma_{\mathrm{ess}}\left(\mathcal{A}_{2}\right)=\left[E_{\min }^{(1,-)}, E_{\max }^{(1,-)}\right] \cup[-\varepsilon,-\varepsilon+2 M] \cup[\varepsilon, \varepsilon+2 M] \cup\left[E_{\min }^{(2,-)}, E_{\max }^{(2,-)}\right] \cup \sigma_{1}^{(+)} \cup \sigma_{2}^{(+)}$, где $E_{\min }^{(1,-)}<-\varepsilon u E_{\max }^{(2,-)}>\varepsilon+2 M$. 
Б3. Если $\alpha \in\left(\alpha_{\max }, \infty\right)$, mo

$$
\sigma_{\mathrm{ess}}\left(\mathcal{A}_{2}\right)=\bigcup_{s= \pm}\left\{\left[E_{\min }^{(1, s)}, E_{\max }^{(1, s)}\right] \cup[s \varepsilon, s \varepsilon+2 M] \cup\left[E_{\min }^{(2, s)}, E_{\max }^{(2, s)}\right]\right\}
$$

где $E_{\min }^{(1, s)}<-\varepsilon, E_{\max }^{(2, s)}>\varepsilon+2 M, s= \pm$.

В. Если выполняется неравенство $\varepsilon>M$, то для любого $\alpha>0$ имеет место соотношение $(-\varepsilon+2 M, \varepsilon) \subset \mathbb{R} \backslash \sigma_{\mathrm{ess}}\left(\mathcal{A}_{2}\right)$.

ДоказАтельство. А. В силу утверждения А леммы 4 при всех значениях $\alpha>0$ оператор $\mathcal{A}_{1}^{(-)}$имеет единственное собственное значение $e_{1}$, лежащее левее $-\varepsilon$. По определению $\sigma_{1}^{(-)}=\left[e_{1}, e_{1}+M\right] \cap(-\infty,-\varepsilon]$, причем $e_{1}<-\varepsilon$. Очевидно, что верно неравенство $E_{\min } \leqslant E_{\min }^{(1,-)}=e_{1}$, поэтому $E_{\min }<-\varepsilon$. Утверждение доказано.

Б1. Пусть $\alpha \in\left(0, \alpha_{\min }\right]$. Тогда в силу следствия 1 оператор $\mathcal{A}_{1}$ имеет единственное простое (изолированное) собственное значение $e_{1}$, которое лежит левее $-\varepsilon$. Как показано выше,

$$
\sigma_{1}^{(-)}=\left[E_{\min }^{(1,-)}, E_{\max }^{(1,-)}\right]=\left[e_{1}, e_{1}+M\right] \cap(-\infty,-\varepsilon],
$$

т. е. $E_{\min }^{(1,-)}=e_{1}$. Кроме того,

$$
\sigma_{1}^{(+)} \cap(-\infty,-\varepsilon)=\varnothing, \quad \sigma_{2}^{(s)} \cap(\varepsilon+2 M, \infty)=\varnothing, \quad s= \pm .
$$

Утверждение доказано.

Б2. Пусть $\alpha \in\left(\alpha_{1}, \infty\right)$. В силу утверждения А леммы 4 оператор $\mathcal{A}_{1}^{(-)}$имеет по одному собственному значению, лежащему левее $-\varepsilon$ и правее $\varepsilon+2 M$. Обозначим их как $e_{1}$ и $e_{2}$ соответственно. Тогда

$$
\begin{aligned}
& \sigma_{1}^{(-)}=\left[E_{\min }^{(1,-)}, E_{\max }^{(1,-)}\right]=\left[e_{1}, e_{1}+M\right] \cap(-\infty,-\varepsilon] \quad \Longrightarrow \quad E_{\min }^{(1,-)}=e_{1}<-\varepsilon, \\
& \sigma_{2}^{(-)}=\left[E_{\min }^{(2,-)}, E_{\max }^{(2,-)}\right]=\left[e_{2}, e_{2}+M\right] \cap[\varepsilon+2 M, \infty) \quad \Longrightarrow \quad E_{\max }^{(2,-)}=e_{2}+M>\varepsilon+2 M .
\end{aligned}
$$

Утверждение доказано.

Б3. Пусть $\alpha \in\left(\alpha_{\max }, \infty\right)$. Тогда в силу следствия 1 оператор $\mathcal{A}_{1}$ имеет по два собственных значения, лежащих левее $-\varepsilon$ и правее $M+\varepsilon$ соответственно. Обозначим их как $e_{1}^{(s)}<-\varepsilon$ и $e_{2}^{(s)}>\varepsilon+M$. По определению множеств $\sigma_{k}^{(s)}, k=1,2$,

$$
\begin{aligned}
& \sigma_{1}^{(s)}=\left[E_{\min }^{(1, s)}, E_{\max }^{(1, s)}\right]=\left[e_{1}^{(s)}, e_{1}^{(s)}+M\right] \cap(-\infty,-\varepsilon] \Longrightarrow E_{\min }^{(1, s)}=e_{1}^{(s)}<-\varepsilon, \\
& \sigma_{2}^{(s)}=\left[E_{\min }^{(2, s)}, E_{\max }^{(2, s)}\right]=\left[e_{2}^{(s)}, e_{2}^{(s)}+M\right] \cap[\varepsilon+2 M, \infty) \Longrightarrow E_{\max }^{(2, s)}=e_{2}^{(s)}+M>\varepsilon+2 M .
\end{aligned}
$$

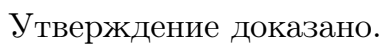

В. Пусть теперь $\varepsilon>M$. Тогда в силу леммы 5 при всех $\alpha>0$ оператор $\mathcal{A}_{1}$ не имеет собственных значений, лежащих в лакуне $(M-\varepsilon, \varepsilon)$. Поэтому справедливо равенство $(-\varepsilon+2 M, \varepsilon) \cap \sigma_{\text {ess }}\left(\mathcal{A}_{2}\right)=\varnothing$. Утверждение и теорема доказаны.

ЗАмЕчАниЕ 4. Если условие (6) не выполняется, т. е. если интегралы в выражении (6) расходятся, то при всех $\alpha>0$ оператор $\mathcal{A}_{1}$ имеет два собственных значения, лежащих левее $-\varepsilon$, и имеет по крайней мере одно собственное значение, лежащее правее $M+\varepsilon$ (см. замечание 3 ). Поэтому

$$
\sigma_{\text {ess }}\left(\mathcal{A}_{2}\right)=\bigcup_{s= \pm}\left\{\left[E_{\min }^{(1, s)}, E_{\max }^{(1, s)}\right] \cup[s \varepsilon, s \varepsilon+2 M]\right\} \cup\left[E_{\min }^{(2,-)}, E_{\max }^{(2,-)}\right] \cup \sigma_{2}^{(+)} .
$$

Здесь $E_{\min }^{(1, s)}<-\varepsilon$ при $s= \pm$ и $E_{\max }^{(2,-)}>\varepsilon+2 M$. 
ЗАмечАниЕ 5. Подчеркнем, что утверждения теоремы 2 в некоторых частных случаях можно получить на основе результатов работы [15], но в отличие от нее в настоящей работе рассмотрены все значения размерности $d$. Леммы 4, 5 и следствие 1 играют ключевую роль при доказательстве теоремы 2 . В случае $d=1$ доказательство конечности дискретного спектра оператора $\mathcal{A}_{2}$ опирается на утверждение А теоремы 2 (см. статью [11]).

Благодарности. Автор выражает глубокую благодарность рецензенту за ценные и полезные замечания, а также благодарит Berlin Mathematical School и Weierstrass Institute for Applied Analysis and Stochastics за приглашение, поддержку и гостеприимство.

\section{Список литературы}

[1] C. Tretter, Spectral Theory of Block Operator Matrices and Applications, Imperial College Press, London, 2008.

[2] H. Spohn, Commun. Math. Phys., 123:2 (1989), 277-304.

[3] M. Hübner, H. Spohn, Ann. Inst. Henri Poincaré, 62:3 (1995), 289-323.

[4] Ю. В. Жуков, Р. А. Минлос, ТМФ, 103:1 (1995), 63-81.

[5] R. A. Minlos, H. Spohn, "The three-body problem in radioactive decay: The case of one atom and at most two photons", Topics in Statistical and Theoretical Physics, American Mathematical Society Translations Ser. 2, 177, eds. R. L. Dobrushin, R. A. Minlos, M. A. Shubin, A. M. Vershik, AMS, Providence, RI, 1996, 159-193.

[6] A.I. Mogilner, "Hamiltonians in solid state physics as multiparticle discrete Schrödinger operators: problems and results", Many-Particle Hamiltonians: Spectra and Scattering, Advances in Soviet Mathematics, 5, ed. R. A. Minlos, AMS, Providence, RI, 1991, 139-194.

[7] К.О. Фридрихс, Возмущения спектра операторов в гилъбертовом пространстве, Мир, М., 1972.

[8] В. А. Малышев, Р. А. Минлос, Линейнъе операторы в бесконечночастичных системах, Наука, М., 1994.

[9] A. E. Lifschitz, Magnetohydrodynamic and Spectral Theory, Developments in Electromagnetic Theory and Applications, 4, Kluwer, Dordrecht, 1989.

[10] B. Thaller, The Dirac Equation, Texts and Monographs in Physics, Springer, Berlin, 1992.

[11] M. Muminov, H. Neidhardt, T. Rasulov, J. Math. Phys., 56:5 (2015), 053507, 24 pp., arXiv: 1410.4763.

[12] Р. Фейнман, Статистическая механика. Курс лекиий, Мир, М., 1978.

[13] С. Н. Лакаев, Т. Х. Расулов, Матем. заметки, 73:4 (2003), 556-564.

[14] S. Albeverio, S. N. Lakaev, T. H. Rasulov, J. Statist. Phys., 127:2 (2007), 191-220.

[15] Т. Х. Расулов, Матем. заметки, 83:1 (2008), 78-94.

[16] И. Ц. Гохберг, М.Г. Крейн, Введение в теорию линейных несамосопряженных операторов, Наука, М., 1965.

[17] М. Рид, Б. Саймон, Методы современной математической физики, т. 4: Анализ операторов, Мир, М., 1982.

Поступила в редакцию 18.01.2015, после доработки 13.04.2015 